\title{
Isolation of Gametophytic Cells and Identification of Their Cell-Specific Markers in Torenia fournieri, T. concolor and Lindernia micrantha
}

\author{
Nao Kawano ${ }^{1}$, Daichi Susaki ${ }^{1}$, Narie Sasaki ${ }^{1}$, Tetsuya Higashiyama ${ }^{1,2}$, \\ and Masahiro M. Kanaoka ${ }^{1 *}$ \\ ${ }^{1}$ Division of Biological Science, Graduate School of Science, Nagoya University, \\ Furo-cho, Chikusa-ku, Nagoya, Aichi 464-8602, Japan \\ ${ }^{2}$ ERATO Live-Holonics Project, JST
}

Received February 1, 2011; accepted March 19, 2011

\begin{abstract}
Summary Gametophytic cells in the female embryo sac, the egg, the central and the synergid cells have unique roles in sexual reproduction in higher plants. To investigate their cell-specific features, an efficient method of distinguishing and isolating each gametophytic cell is required. By testing the activities of several cellulase enzymes, we modified our previous cell isolation method and succeeded in isolating each cell from the Torenia and Lindernia species. We also established genetic markers that show specific expression patterns in each gametophytic cell. These markers, together with the cell isolation method, will be useful for analyzing the function of gametophytic cells.
\end{abstract}

Key words Egg cell, Central cell, Synergid cell, Protoplast isolation, Gametophytic cell-specific markers, Torenia.

Sexual reproduction is a unique feature of higher angiosperms. The embryo sac, which contains 1 egg cell, 1 central cell, 2 synergid cells, and 3 antipodal cells, is the female gametophytic organ required for the process of sexual reproduction in plants (Drews and Yadegari 2002). The egg cell is fertilized by a sperm cell to generate an embryo, while the central cell is fertilized by another sperm cell to generate an endosperm. The synergid cells are required for micropylar pollen tube guidance (Higashiyama et al. 2001, Kasahara et al. 2005). Given that the embryo sac is deeply embedded in the ovule, knowledge of the genes involved in its development or its function remains limited.

A variety of methods, including osmotic stress, mechanical force, and cellulase enzyme treatments, have been attempted to isolate gametophytic cells from the ovule to analyze their features (Theunis et al. 1991, Huang and Russell 1989, Katoh et al. 1997, Kovacs et al. 1994). Enzyme treatment in particular has been used to generate protoplasts from the female gametophytes of a wide range of plant species including Ginkgo biloba, Salvinia natans, Laminaria digitata, Petunia hybrida (Laurain et al. 1993, Nakamura and Maeda 1994, Benet et al. 1997, Sangthong et al. 2009), and Torenia fournieri (Mol 1986, Chen et al. 2008).

Torenia fournieri is a unique plant species that exhibits protruding embryo sacs (Higashiyama et al. 1998). Given this feature, female gametophytic cells are easily accessed when the carpel wall is dissected and the ovules are removed from the ovary (Higashiyama et al. 2001, Higashiyama 2002). Previously, we succeeded in generating a cDNA library from 25 synergid protoplasts isolated by cellulase treatment and identified genes specifically expressed in the synergid cells that functioned as pollen tube attractants (Okuda et al. 2009). These results suggested that gene expression analysis from isolated cells is a powerful tool to investigate the function of gametophytic

*Corresponding author, e-mail: mkanaoka@bio.nagoya-u.ac.jp 
cells. However, since only 1 egg cell, 1 central cell, and 2 synergid cells exist in an ovule, the total number of obtainable cells from one treatment is limited. Longer enzyme treatment may help isolate a larger number of cells, but may affect the cellular motility and thus the gene expression patterns within the cells (Leduc et al. 1995). For these reasons, improving the isolation method for collecting large numbers of cells in a short period of time is required.

Recently, large-scale analyses to identify the genes expressed in specific gametophytic cells have been undertaken using Oryza sativa and Arabidopsis thaliana. Specifically, intact egg and synergid cells were isolated from the unfertilized ovaries of $O$. sativa by manual manipulation without using enzymes (Ohnishi et al. 2010). A differential expression screening between the wild type and determinant infertilel (difl) mutant, which lacks a functional embryo sac, identified several genes expressed in Arabidopsis female gametophytes (Steffen et al. 2007). By examining gene expression in myb98, which is defective for the synergid cell-specific transcription factor MYB98, genes specifically expressed in the synergid cell that functioned under MYB98 were identified (Punwani et al. 2007). For Arabidopsis, a laser-associated microdissection method was used to dissect the cells of the mature female gametophyte with minute cross-sections, and genes specifically expressed in each cell type were identified (Wuest et al. 2010). Genes that shared similar expression profiles even across monocot and eudicot species were regarded as cell-specific markers common to a wide range of plant species.

Here we compared cellulase enzymes for protoplast isolation activities and report a more efficient method of isolating gametophytic protoplasts from 2 additional plant species, Torenia concolor and Lindernia micrantha. We also investigated the genes specifically expressed in all types of gametophytic cells. We found that some genes showed cell-type-specific expression in $T$. fournieri, T. concolor, and L. micrantha, suggesting that these genes would be good markers for the cell types.

Materials and methods

\section{Plant growth conditions}

Torenia fournieri cv. Blue \& White and T. concolor were grown in a plant growth room under stable temperature $\left(25^{\circ} \mathrm{C}\right)$ and continuous light conditions. Lindernia micrantha was grown in the growth chamber (NK System) under stable temperature $\left(25^{\circ} \mathrm{C}\right)$ and cycled light conditions (16h light and $8 \mathrm{~h}$ dark).

\section{Isolation of gametophytic cells}

The flowers of $T$. fournieri and T. concolor $2 \mathrm{~d}$ after blooming were used for isolating cells. Because L. micrantha is an auto-pollinated self-compatible species, flowers were emasculated $1 \mathrm{~d}$ prior to the experiment.

Isolation of gametophytic cells was conducted as described previously with some modifications (Okuda et al. 2009). The cellulase enzyme solution contained 1\% (w/v) cellulase, $0.3 \%$ macerozyme RS (Yakult), $0.05 \%$ pectolyase Y-23 (Yakult), $5 \mathrm{mM} \mathrm{CaNO}_{3}$, and $0.4 \mathrm{M}$ mannitol. Three types of cellulase, CELF (Worthington), the cellulase from Aspergillus (Sigma), and that from Trichoderma (Sigma), were used to compare enzyme activities. Aliquots of the cellulase solutions were stored at $-80^{\circ} \mathrm{C}$ until used.

The cellulase enzyme solution was warmed to $28^{\circ} \mathrm{C}$ just before use. A mature ovary was dissected and the ovules were placed in $300 \mu \mathrm{l}$ of the cellulase enzyme solution. These were maintained at $28^{\circ} \mathrm{C}$ for $1 \mathrm{~h}$ to generate protoplasts. Cells were distinguished by their morphological features and the quality of the protoplasts was evaluated by observation under an Olympus IX71 inverted microscope. Protoplasts were collected using a PicoPipet (Altair Co., Japan), then rinsed twice with $100 \mu \mathrm{l}$ of wash solution $\left(5 \mathrm{mM} \mathrm{CaNO}_{3}\right.$ and $0.4 \mathrm{M}$ mannitol). After washing, they were 
collected by brief centrifugation and frozen with liquid $\mathrm{N}_{2}$ to isolate RNA.

Estimation of enzyme activities for protoplast isolation

To evaluate the enzyme activities for protoplast isolation, first, the ovules were taken from the ovary as described above. Because the protruding embryo sacs might be destroyed during this process, the number of ovules with intact embryo sacs was counted. Next, the ovules were treated with enzyme solution at $28^{\circ} \mathrm{C}$. The number of central cells detached from the ovules was counted every 30 min during the treatment. The enzyme activity was defined as the rate of the number of detached central cells versus ovules with intact embryo sacs.

\section{DNA staining}

Protoplasts were rinsed with wash solution, then stained with 1\% SYTO-11 (Invitrogen) in the wash solution for $1 \mathrm{~min}$. Green fluorescence was observed under an Olympus IX71 epifluorescence microscope.

\section{Isolation of RNA from protoplasts and RT-PCR}

Total RNA from isolated egg and central and synergid cell protoplasts was extracted using Dynabeads (Invitrogen) according to the manufacturer's instructions. cDNA was synthesized using SuperScriptIII (Invitrogen) reverse transcriptase, and $250 \mathrm{ng}$ of cDNA was used as a template for each PCR. The sequences of the primers used for RT-PCR were as follows (from $5^{\prime}$ to $3^{\prime}$ ): $D D 45$, CAAGTCGTGTAGTAGCGAAG and CACAAAACCCTCGAAGAAC; $S G S 3$, GTTTGGCCTCCTATGGTG and CTCACGATGCCAAAAGTC; $S W N$, CCATAGCGGTAGTCATAAAAG and CTTGTTTTGCAGCTGGAC. CRP1, CRP4, and GAPDH were as previously described (Okuda et al. 2009). We used 35 PCR cycles to amplify CRP1 and CRP4, while 50 cycles were run for the other markers.

\section{Results and discussion}

We compared the gametophytic cell features between $T$. fournieri and the related species $T$. concolor and L. micrantha (Fig. 1). These species show similar floral morphologies to T. fournieri

\section{T. fournieri}
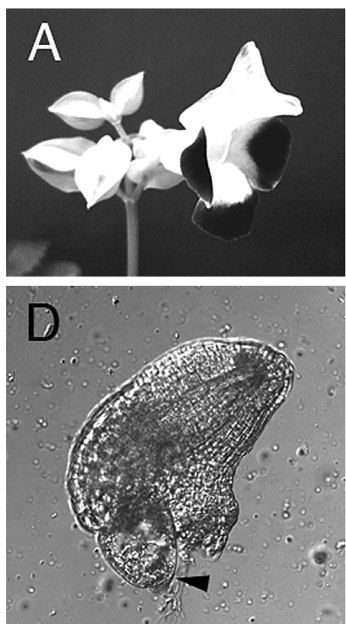
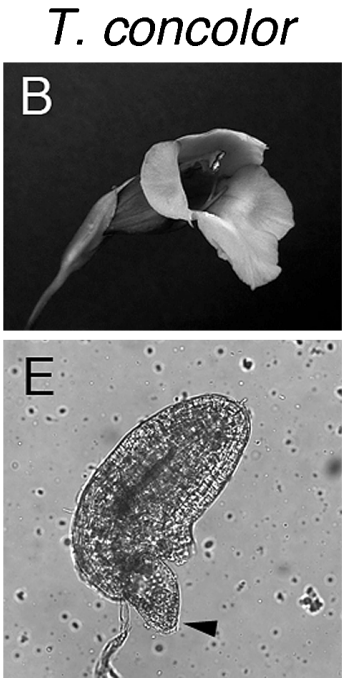

\section{L. micrantha}
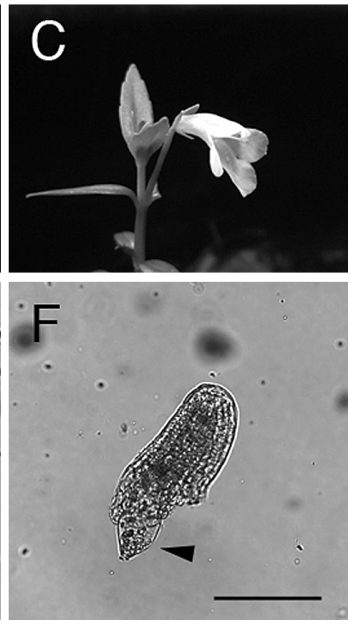

Fig. 1. Flower and ovule morphology of the plant species used in this study. Flowers of T. fournieri (A), T. concolor (B), and L. micrantha (C). Ovules of T. fournieri (D), T. concolor (E), and L. micrantha (F) had protruding embryo sacs (arrowheads). Scale bar shown in F, $100 \mu \mathrm{m}$ for (D-F). 
(Figs. 1A-C) and exhibit embryo sacs protruding from the ovules (Figs. 1D-F). We attempted to isolate gametophytic cells via treatment with cellulase. We previously reported that the synergid cells of T. fournieri could be isolated using CELF cellulase (Okuda et al. 2009). However, we found that this enzyme was not sufficient for cell isolation from $T$. concolor and L. micrantha (Table 1). Moreover, the central cell, located at the innermost position among these 3 types of gametophytic cells, did not detach by cellulase treatment in these 2 species (Table 1, Fig. 2D). Therefore, we attempted to compare the activities of several commercially available cellulases. Aspergillus cellulase was ineffective at isolating the central cells of all 3 plant species, even after $2 \mathrm{~h}$ of treatment (Figs. 2A, E, Table 1). In contrast, the cellulase from Trichoderma worked well (Figs. 2B-C, F, Table 1). The central cells were isolated from more than $40 \%$ of $T$. fournieri embryo sacs after $1.5 \mathrm{~h}$ of treatment, which is a higher efficiency than that of CELF (Table 1). Even after $1 \mathrm{~h}$ of treatment, $7.8 \%$ of $T$. fournieri central cells were detached. These results suggest that the cellulase

Table 1. Comparison of cellulase activities for gametophytic cell isolation

\begin{tabular}{|c|c|c|c|c|c|}
\hline \multirow{2}{*}{ Cellulase } & \multirow{2}{*}{ Plant species } & \multicolumn{4}{|c|}{$\%$ of central cells detached from the ovules (central cells/ovules) } \\
\hline & & $0.5 \mathrm{~h}$ & $1.0 \mathrm{~h}$ & $1.5 \mathrm{~h}$ & $2.0 \mathrm{~h}$ \\
\hline \multirow[t]{3}{*}{ CELF } & T. fournieri & $0.0(0 / 347)$ & $0.0(0 / 347)$ & $0.29(1 / 347)$ & $1.2(4 / 347)$ \\
\hline & T. concolor & $0.0(0 / 549)$ & $0.0(0 / 549)$ & $0.0(0 / 549)$ & $0.0(0 / 549)$ \\
\hline & L. micrantha & $0.0(0 / 121)$ & $0.0(0 / 121)$ & $0.0(0 / 121)$ & $0.0(0 / 121)$ \\
\hline \multirow[t]{3}{*}{ Aspergillus } & T. fournieri & $0.0(0 / 205)$ & $0.0(0 / 205)$ & $0.0(0 / 205)$ & $0.0(0 / 205)$ \\
\hline & T. concolor & $0.0(0 / 290)$ & $0.0(0 / 290)$ & $0.0(0 / 290)$ & $0.0(0 / 290)$ \\
\hline & L. micrantha & $0.0(0 / 301)$ & $0.0(0 / 301)$ & $0.0(0 / 301)$ & $0.0(0 / 301)$ \\
\hline \multirow[t]{3}{*}{ Trichoderma } & T. fournieri & $0.0(0 / 462)$ & $7.8(36 / 461)$ & $41.9(83 / 198)$ & $50.5(257 / 509)$ \\
\hline & T. concolor & $0.0(0 / 1252)$ & $1.8(23 / 1252)$ & $5.0(62 / 1252)$ & $8.5(106 / 1252)$ \\
\hline & L. micrantha & $0.0(0 / 125)$ & $0.0(0 / 125)$ & $1.6(2 / 125)$ & $5.6(7 / 125)$ \\
\hline
\end{tabular}
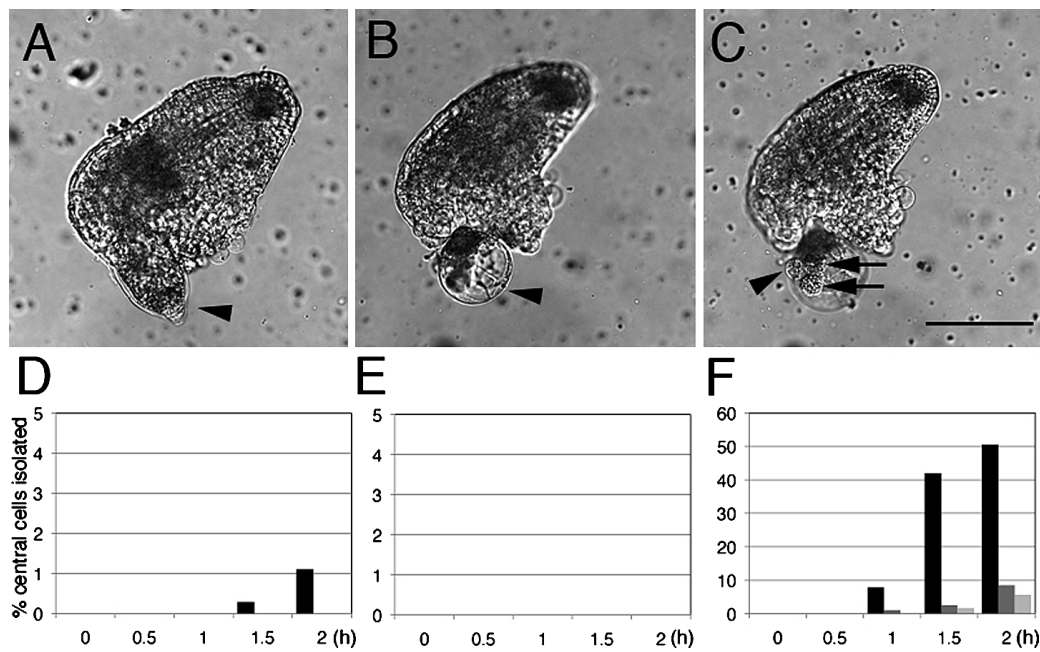

Fig. 2. Effects of cellulases for protoplast isolation. Gametophytic cells did not detach from the embryo sac through treatment with the cellulase from Aspergillus (A, arrowhead), whereas following treatment with cellulase from Trichoderma, the central cell (B, arrowhead), the egg cell (C, arrowhead) and the synergid cells $(\mathrm{C}$, arrows) became protoplasts. Cellulase activities for protoplast isolation by CELF (D), the cellulase from Aspergillus (E), and the cellulase from Trichoderma (F) were measured after $0.5,1,1.5$, and $2 \mathrm{~h}$ of treatment. The Y-axis represents the isolation rate of the central cell. The black, dark gray, and pale gray bars represent T. fournieri, T. concolor, and $L$. micrantha, respectively. Scale bar, $20 \mu \mathrm{m}$. 
from Trichoderma can be used to isolate gametophytic cells in Torenia.

To isolate and collect the protoplasts of gametophytic cells, we treated ovules with Trichoderma cellulase (Fig. 3). The egg, and central and synergid cells of T. fournieri exhibited unique morphological features (Figs. 3A-C). The central cell was largest and exhibited 1 large vacuole (Fig. 3B). The egg cell and the synergid cells were similar in size, but numerous cytoplasmic strands and small vacuoles distinguished the egg (Fig. 3A), whereas the synergid cells had 1 large vacuole (Fig. 3C). The isolated protoplasts often appeared fused; thus, we tested whether they were from single cells by staining the cells with the DNA-binding dye SYTO-11,

\section{Egg cell}
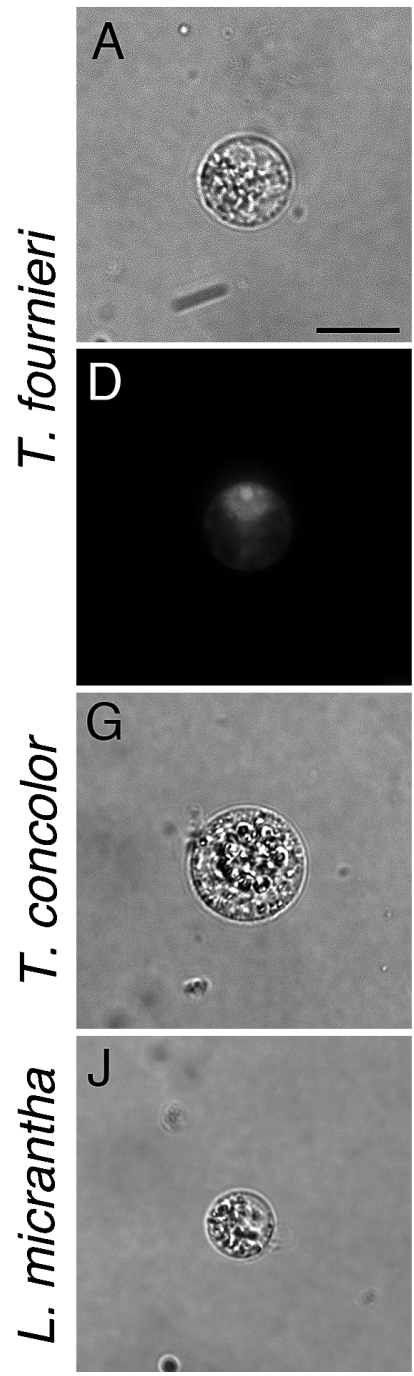

\section{Central cell Synergid cell}
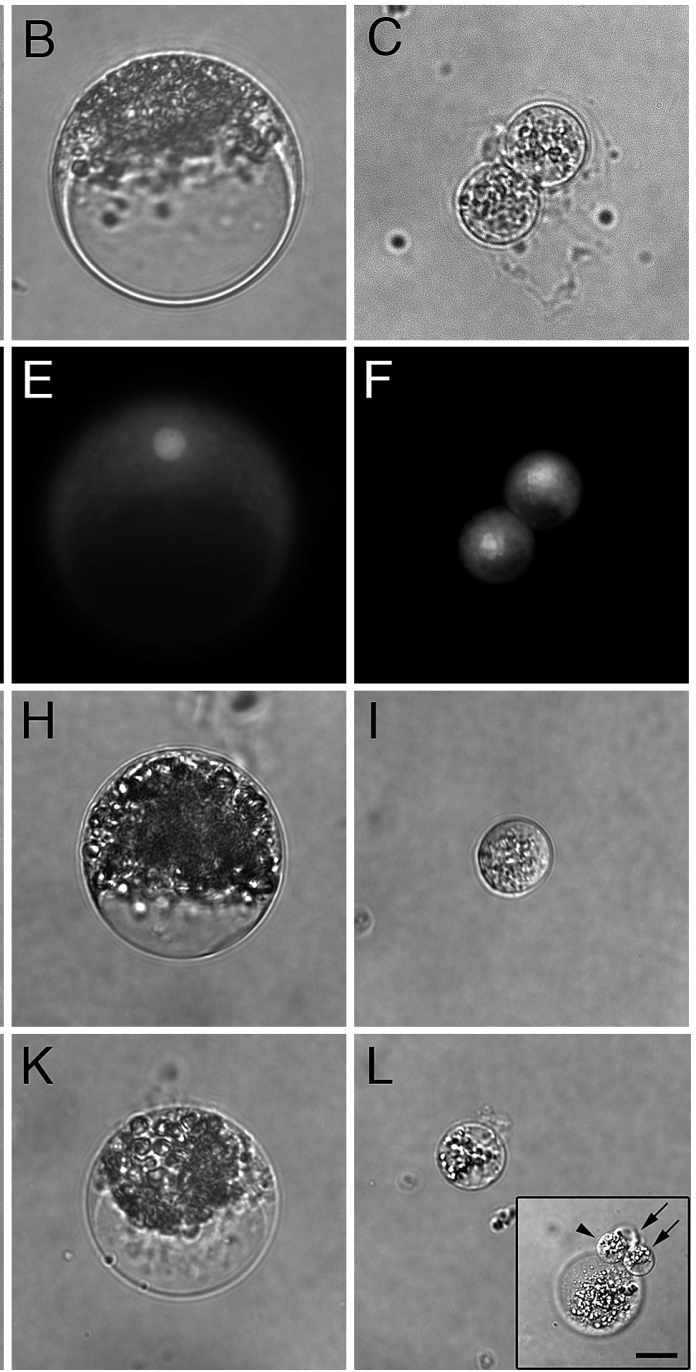

Fig. 3. Isolated protoplasts from 3 plant species. Protoplasts of the egg cell (A), the central cell (B), and the synergid cells (C) from $T$. fournieri. The same cells were stained with CYTO-11 to visualize DNA (D-F). Nuclei were identified as bright spots. Protoplasts of the egg cell (G), the central cell (H), and the synergid cells (I) from T. concolor. Protoplasts of the egg cell (J), the central cell (K) and the synergid cells (L) from L. micrantha. Inset in (L), a cluster of protoplasts. One egg cell (arrowhead), 2 synergid cells (arrows), and one central cell (large cell) were detached from the same ovule. Scale bar shown in (A), $20 \mu \mathrm{m}$ for (A-L); in the inset of (L), $20 \mu \mathrm{m}$. 
which rapidly permeates living protoplasts (Bethke et al. 1999). We found that all of the isolated protoplasts had individual nuclei, indicating they were from single cells and that the cells had not fused (Figs. 3D-F). We also isolated protoplasts from T. concolor and L. micrantha (Figs. 3G-L). In both species, the central cells were large and easily distinguished. To distinguish the egg cell and the synergid cells, the cells were carefully detached and isolated from the cluster of detached gametophytic cells (Fig. 3L, inset), and the cell types were subsequently confirmed by marker gene expression levels (see below).

Next, we isolated mRNA from each type of $T$. fournieri cell. We investigated the expression patterns of the following cell-specific marker genes found in A. thaliana: DD45, which encodes a cysteine-rich polypeptide and is expressed specifically in the egg cell (Steffen et al. 2007); SGS3 and $S W N$, which encode the SUPPRESSOR OF GENE SILENCING3 protein required for posttranscriptional gene silencing; and a Polycomb group protein SWINGER expressed in the central cell (Mourrain et al. 2000, Chanvivattana et al. 2004). A putative homolog of DD45 in T. fournieri was specifically found in the cDNA template from the egg cell (Fig. 4, left row). $S G S 3$ and $S W N$ were specifically expressed in the central cell (Fig. 4, middle row), suggesting that these genes could be used as egg cell- and central cell-specific markers in Torenia. As previously reported, CRPI and $C R P 4$, which encode cysteine-rich polypeptides, were specifically expressed in the synergid cell (Fig. 4, right row) (Okuda et al. 2009). Next, we tested these markers using the cDNA templates from T. concolor and L. micrantha (Fig. 4). In both species, the RT-PCR products from these markers were specifically found in each cell type. These results suggest that we successfully isolated protoplasts from each type of cell with no contamination from other gametophytic cells.

Our results indicate that cellulases differ in their cell isolation activities. Given that longer enzyme treatment could harm the cells, while shorter treatment times would more closely mimic natural conditions (Leduc et al. 1995), our modified method using the cellulase from Trichoderma will be especially useful for the rapid collection of large amounts of high-quality cells and mRNAs for future analysis of gene expression profiles in gametophytic cells. The comparison of gene expression profiles of Torenia gametophytic cells with those of eudicot species such as $A$. thaliana (Steffen et al. 2007, Wuest et al. 2010) and monocot species such as O. sativa (Ohnishi et al. 2010) will reveal the common and distinct features of plant gametophyte genes.

We showed that putative homologous genes of the Arabidopsis gametophytic cell-specific genes, $D D 45, S G S 3$, and $S W N$, were also specifically expressed in the corresponding types of cells in Torenia and Lindernia. This is the first report of egg cell and central cell markers in these plant

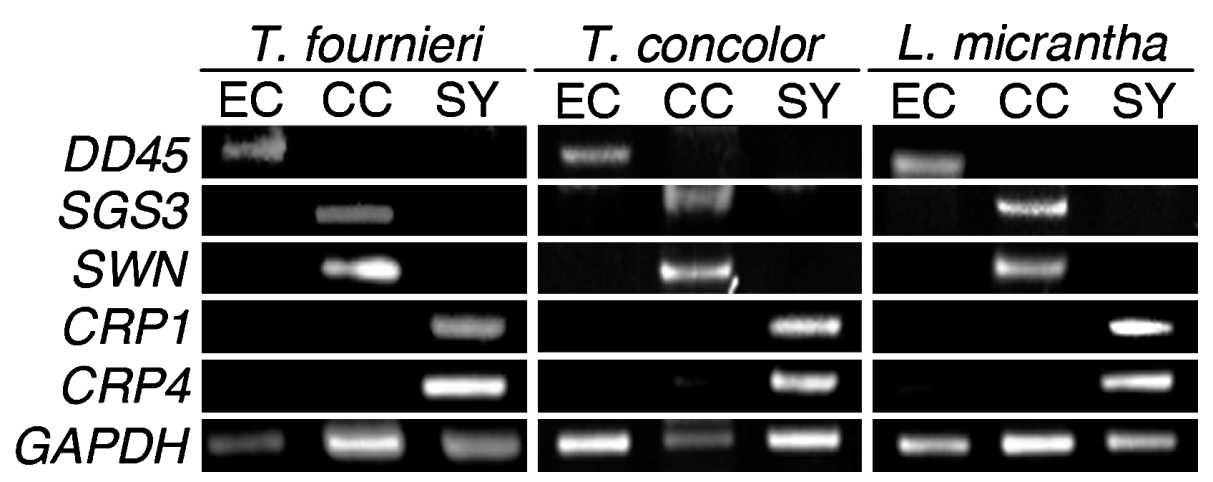

Fig. 4. Cell-specific expression of marker genes. cDNAs generated from an isolated egg cell (EC), central cell (CC), and synergid cell (SY) from T. fournieri (left row), T. concolor (middle), and L. micrantha (right) were used as the templates for RT-PCR. DD45 was used as an egg cell marker, and $S G S 3$ and $S W N$ were central cell markers. CRP1 and CRP4 were synergid cell markers. GAPDH (glyceraldehyde 3-phosphate dehydrogenase) showed ubiquitous expression in all cell types. 
species. Together with our previously reported synergid cell-specific genes, CRP1 and CRP4, these will be good markers for gametophytic cells. In addition, this information will be useful for generating constructs that enable us to express or downregulate genes of interest in a gametophyte cell-specific manner.

\section{Acknowledgments}

We are grateful to Goro Kokubugata, Hiroshi Yamashita, Nobuhide Kato, Shigehiro Sagawa and Sachiko Nishida for plant materials, Yoshiyuki Matsubara for technical assistance, and Yoko Mizuta for helpful discussions. This work was supported by Grants from the Ministry of Education, Culture, Sports, Science and Technology, Japan (nos. 18075004 and 19370017 to T.H.; no. 18GS0314-01 to N.S.; nos. 20870020 and 21770041 to M.M.K.), the Japan Society for the Promotion of Science for Young Scientists (to D.S.), the Japan Science and Technology Agency (PRESTO project to T.H.), the Yamada Science Foundation (to T.H.), and the Mitsubishi Foundation (to T.H.).

\section{References}

Benet, H., Gall, E. A., Asensi, A. and Kloareg, B. 1997. Protoplast regeneration from gametophytes and sporophytes of some species in the order Laminariales (Phaeophyceae). Protoplasma 199: 39-48.

Bethke, P. C., Lonsdale, J. E., Fath, A. and Jones, R. L. 1999. Hormonally regulated programmed cell death in barley aleurone cells. Plant Cell. 11: 1033-1046.

Chanvivattana, Y., Bishopp, A., Schubert, D., Stock, C., Moon, Y. H., Sung, Z. R. and Goodrich, J. 2004. Interaction of Polycomb-group proteins controlling flowering in Arabidopsis. Development 131: 5263-5276.

Chen, S. H., Ang, Y. H., Liao, J. R., Kuang, A. X. and Tian, H. Q. 2008. Isolation of egg cells and zygotes of Torenia fournieri L. and determination of their surface charge. Zygote 16: 179-186.

Drews, G. N. and Yadegari, R. 2002. Development and function of the angiosperm female gametophyte. Annu. Rev. Genet. 36: 99-124.

Higashiyama, T. 2002. The synergid cell: Attractor and acceptor of the pollen tube for double fertilization. J. Plant Res. 115: $149-160$.

- Kuroiwa, H., Kawano, S. and Kuroiwa, T. 1998. Guidance in vitro of the pollen tube to the naked embryo sac of Torenia fournieri. Plant Cell. 10: 2019-2032.

—, Yabe, S., Sasaki, N., Nishimura, Y., Miyagishima, S., Kuroiwa, H. and Kuroiwa, T. 2001. Pollen tube attraction by the synergid cell. Science 293: 1480-1483.

Huang, B. Q. and Russell, S. D. 1989. Isolation of Fixed and Viable Eggs, Central Cells, and Embryo Sacs from Ovules of Plumbago-Zeylanica. Plant Physiol. 90: 9-12.

Kasahara, R. D., Portereiko, M. F., Sandaklie-Nikolova, L., Rabiger, D. S. and Drews, G. N. 2005. MYB98 is required for pollen tube guidance and synergid cell differentiation in Arabidopsis. Plant Cell. 17: 2981-2992.

Katoh, N., Lorz, H. and Kranz, E. 1997. Isolation of viable egg cells of rape (Brassica napus L.). Zygote 5: 31-\&.

Kovacs, M., Barnabas, B. and Kranz, E. 1994. The Isolation of Viable Egg Cells of Wheat (Triticum-Aestivum L.). Sex. Plant Reprod. 7: 311-312.

Laurain, D., Chenieux, J. C. and Tremouillauxguiller, J. 1993. Direct Embryogenesis from Female Haploid Protoplasts of Ginkgo-Biloba L., a Medicinal Woody Species. Plant Cell Rep. 12: 656-660.

Leduc, N., Matthysrochon, E. and Dumas, C. 1995. Deleterious Effect of Minimal Enzymatic Treatments on the Development of Isolated Maize Embryo Sacs in Culture. Sex. Plant Reprod. 8: 313-317.

Mol, R. 1986. Isolation of Protoplasts from Female Gametophytes of Torenia-Fournieri. Plant Cell Rep. 5: $202-206$.

Mourrain, P., Beclin, C., Elmayan, T., Feuerbach, F., Godon, C., Morel, J. B., Jouette, D., Lacombe, A. M., Nikic, S., Picault, N., Remoue, K., Sanial, M., Vo, T. A. and Vaucheret, H. 2000. Arabidopsis SGS2 and SGS3 genes are required for posttranscriptional gene silencing and natural virus resistance. Cell 101: 533-542.

Nakamura, M. and Maeda, M. 1994. Isolation and Culture of Protoplasts from Young Sporophytes of Salvinia-Natans Aseptically Obtained by Coculture of Female and Male Gametophytes. Plant Cell Tissue Organ Cult. 36: 237-242.

Ohnishi, T., Takanashi, H., Mogi, M., Takahashi, H., Kikuchi, S., Yano, K., Okamoto, T., Fujita, M., Kurata, N. and Tsutsumi, N. 2010. Distinct Gene Expression Profiles in Egg and Synergid Cells of Rice as Revealed by Cell Type-specific Microarrays. Plant Physiol. 155: 881-891. 
Okuda, S., Tsutsui, H., Shiina, K., Sprunck, S., Takeuchi, H., Yui, R., Kasahara, R. D., Hamamura, Y., Mizukami, A., Susaki, D., Kawano, N., Sakakibara, T., Namiki, S., Itoh, K., Otsuka, K., Matsuzaki, M., Nozaki, H., Kuroiwa, T., Nakano, A., Kanaoka, M. M., Dresselhaus, T., Sasaki, N. and Higashiyama, T. 2009. Defensin-like polypeptide LUREs are pollen tube attractants secreted from synergid cells. Nature 458: 357-361.

Punwani, J. A., Rabiger, D. S. and Drews, G. N. 2007. MYB98 positively regulates a battery of synergid-expressed genes encoding filiform apparatus localized proteins. Plant Cell. 19: 2557-2568.

Sangthong, R., Chin, D. P., Supaibulwatana, K. and Mii, M. 2009. Gametosomatic hybridization between egg cell protoplast and mesophyll protoplast of Petunia hybrida. Plant Biotechnol. J. 26: 377-383.

Steffen, J. G., Kang, I. H., Macfarlane, J. and Drews, G. N. 2007. Identification of genes expressed in the Arabidopsis female gametophyte. Plant J. 51: 281-292.

Theunis, C. H., Pierson, E. S. and Cresti, M. 1991. Isolation of Male and Female Gametes in Higher-Plants. Sex. Plant Reprod. 4: 145-154.

Wuest, S. E., Vijverberg, K., Schmidt, A., Weiss, M., Gheyselinck, J., Lohr, M., Wellmer, F., Rahnenfuhrer, J., von Mering, C. and Grossniklaus, U. 2010. Arabidopsis Female Gametophyte Gene Expression Map Reveals Similarities between Plant and Animal Gametes. Curr. Biol. 20: 506-512. 